\title{
Il regno doloroso di Paolo Valesio.
}

\section{Mario Lunetta}

In quel romanzo a forte caratura allegorica che è L'ospedale di Manhattan (1978), prima e perentoria prova del Valesio narratore, ci sono due passi almeno che non possono non essere considerati un introibo, perfino liturgico (insieme linguistico e teorico-ideologico), all'appena apparso Regno doloroso (Milano: Spirali Edizioni, 1983). Cosí suona il primo, che conclude la Pre-Fazione dell'autore: "Si può dire qui soltanto che il linguaggio del romanzo . . è un linguaggio che non irride se stesso, e tenta di non impoverirsi demagogicamente, per apparire a basso prezzo spregiudicato. Dunque, ancora una volta mobilitazione totale (nei limiti, s'intenda, delle forze di chi scrive). Diacronicamente: recupero dell'antica e grande tradizione italiana (di sintassi cosí come di lessico). Nella sincronia: rottura di ogni purismo per accostare liberamente alle antiche native parole - calchi da altre lingue (soprattutto l'inglese e il francese moderni). Dimostrando fra l'altro la validità di un'intuizione antica (del Castiglione, a cagion d'esempio): che la lingua italiana può agilmente accogliere tutti i contributi stranieri, senza snaturarsi - al contrario, arricchendo le proprie connotazioni, la propria capacità di penetrare il mondo, conoscerlo, descriverlo." E cosí suona il secondo, molto complementarmente: "Basta con le grottesche letterarie sul Nulla e sulla tragedia dell'Incomunicabilità! Occorre costringersi, quasi stringendo forte le braccia intorno al proprio petto per rattenersi da ogni tergiversazione, fuga, divagazione - basta costringersi a una interrogazione vera di se stessi, anche se dolorosa. In questo esame che ravviva un antico senso della parola esame (quello di 'tortura'), lo scrittore non può non perdere il suo equilibrio, la sua stazione erta sullo scanno (è il prezzo che deve pagare): e distolga pure il viso con una smorfia appenata, come se il suo stilo invece di toccare e punteggiare il foglio gli ferisse la mano. La penna - può esservi dubbio? - è lo strumento di ferite per eccellenza, e la paranomastica tensione fra questa parola e la consimile (pena) è antica già ai tempi in cui la Stampa riprende (dal Petracco, da altri), e ostenta, questo rapporto in cui il piacere è sempre nuovo (e ammoderna le armi del dio antico - dalle frecce alle bocche da

QUADERNI d'italianistica, Volume IV, No. 2, 1983 
fuoco; cosí da mostrare come essa avrebbe veduto con freschi occhi, o colto, la sordida bellezza e la minaccia offerte dalle armi primitive degli stracciati):

S'Amor con novo insolito focile,

Ov'io non potea gir, m'alzò a tal loco,

Perché non può con non usato gioco

Far la penna e la pena in me simile

Gli stiramenti addolorati del viso sono uno dei pochi segni sicuri della nostra fratellanza. Cosí almeno sembra a me, che quasi sempre trovo alieni o minacciosi i volti dei miei simili. E infatti: permangono seri? Mi paiono, allora, direttamente ostili. Sorridono? Ancora mi sembrano aggressivi, pieni di non espresse ironie ed allusioni. Ma se smorfiano - per un attimo, di dolore, e la loro pelle si stira sugli zigomi - ah i suoi zigomi, ognuno trova la bellezza in luoghi diversi, per me quello spazio era la parte cruciale, anche se il mero atto di citare (ma io mi consento il lusso di addirittura laudare) questi spazi esigui, questi non codificati attributi, rischia di apparire come un'intollerabile insolenza, ai nostri cuori stretti di cauti parlatori/scrittori moderni. Se la loro pelle si stira sugli zigomi, e si risucchia intorno alle labbra, io li riscopro fratelli, e sorelle senza calore di sesso." A pag. 111 de Il regno doloroso, a proposito dello sguardo di una donna poeta, è scritto: "in quell'occhiata ingenuamente aperta essa ha rivelato uno dei pochi tratti redentivi del, e della poeta: il coraggio di sfidare l'ingenuità."

Ciascuna di queste citazioni potrebbe figliarne altre dieci: e ne risulterebbe ad ogni modo un ben sapido assemblaggio. Ma ritengo comunque, le già esibite, sufficienti à foison per una definizione delle intenzionalità di un autore come Paolo Valesio, impegnato fin dal primo romanzo a recuperare attivamente, nella scrittura inventiva, le sue sofisticatissime risorse e esperienze di linguista: ut vidimus. Magari, e non poco scandalosamente, avanzando un'ipotesi di elogio della Restaurazione in ordine alla lingua e alla sua ricchezza - coincidendo su questo terreno con le posizioni espresse dal sottoscritto in un'occasione non lontana (v. Il Ponte, novembre-dicembre 1982), nei termini che seguono - e mi si perdoni l'autocitazione, che però ritengo in una qualche misura appropriata e magari utile a un dibattito da riaprirsi (ancora! ancora! c'è da rabbrividire) sulla lingua, con riferimento alla sempre piú massiccia serie di crimini perpetrati contro l'italiano, il suo spessore storico, la sua disarmonia prestabilita: "Oggi il compito primario di uno scrittore degno del nome è, paradossal- 
mente, quello di un addetto alla 'restaurazione' della lingua. È questo il compito piú rivoluzionario a cui egli possa attendere: difendere la ricchezza, la profondità, l'imprevedibilità incontrollabile della lingua; la sua ambiguità che è complessità; la sua dépense insomma - direbbe Bataille - contro la taccagneria interessata e usuraia dei risparmiatori editoriali e televisivi, per i quali tutti i linguaggi complessi devono essere ridotti a linguaggi semplici: cioè a balbuzie, a pre-afasia." Proprio in quella "mobilitazione totale," in quel "recupero dell'antica e grande tradizione italiana (di sintassi cosí come di lessico)" di cui si discute in limine all'Ospedale di Manhattan riposa lo sperimentalismo rimosso di un romanzo come Il regno doloroso. Di piú: il segno di questa rimozione è una traccia, appunto, dolorosa: la pena provocata dall'incisione della penna sul foglio, cioè sulla pelle dello scrittore. Si ha quasi vergogna a sottolineare l'assoluta assenza di connotazioni in qualche modo connesse col concetto di peccato, di sofferenza e di risarcimento oltremondano, nel titolo del secondo romanzo valesiano. $\mathrm{E}$ d'altro canto, è pressoché inevitabile indicarne la connotazione (consapevole) di insufficiente rapporto con la pienezza del vivere, che è lo stemma dominante del nostro tempo indigente, direbbe Heidegger. In esergo campeggia una riflessione di Ralph Waldo Emerson: "È strano come sia doloroso il mondo reale - il regno doloroso del tempo e dello spazio." E non pretendeva il Valesio dell'Ospedale di Manhattan, come ho già ricordato, che occorre "costringersi a una interrogazione vera di se stessi, anche se dolorosa"? Grande, la coerenza e la coibenza dei due romanzi di Valesio. Grande e fatale: anche nel senso che l'allegorismo di "taglia forte" del primo viene a sfociare nell'allegorismo "di taglia esile" del secondo, senza nulla perdere di acuzie polisensa, anzi forse guadagnandoci in sottigliezza stilistica: e probabilmente scendendo - con un'operazione di sostituzione d'effetto, potremmo dire: cioè l'ironia del Regno al posto del sarcasmo dell'Ospedale - a livelli di scandaglio magari piú esigenti, in cui la posta della caccia è la totalità di un se stesso che si confronta senza tregua con la propria immagine allo specchio: e la propria immagine è la bellezza dolorosa dei suoi simili specialmente in figura femminile.

Si è visto come per Valesio uno dei pochi "tratti redentivi" del poeta consista nel "coraggio di sfidare l'ingenuità." Appunto: la diàtesi dello scrittore è, ora, di specie minimalistica. Il suo occhio indaga e isola il dettaglio. La sua voce parla sommesso: tanto piú quanto piú folgorante e clamoroso è il fotogramma capace di sprigionare luce simbolica. La Spaltung ora non si verifica piú tra Nuovo Mondo e Vecchio Mondo, macrospezzoni che la coscienza 
critica di un intellettuale europeo cerca (quasi disperatamente) di far combaciare, a dispetto di tutto, e prima di tutto a dispetto di quelle che sono, davvero e irrimediabilmente due culture - "le due culture," si diceva un ventennio fa, con scolastica banalità, pensando a quella umanistica e a quella scientifica. . . . Ora, nel Regno, la scissione è forse piú irrimediabile, proprio in quanto si produce all'interno dell'unità coscienziale dei personaggi raccontati: $i d$ est, di colui che racconta, ombra invisibile eppure, paradossalmente, Narratore Onnisciente. Il fatto è che in quel libro di straordinario valore che è $I l$ regno doloroso, non c'è neanche la memoria dell'ideologia inclusa nella mimesi naturalistica, eppure - contraddizione che è verità poetica - la voce che registra gli eventi e le evenienze è quella di un Osservatore situato "al vertice": sulla sommità del mondo: nell'acropoli del Regno, diciamo pure. Ma la sua è al tempo stesso, una presenza ingenua e supremamente intelligente, che costruisce la sua città nel mentre la vede e la descrive.

Come, quindi, la vede, la costruisce e la descrive? Nell'unico modo possibile, qui e ora, a chi abiti la scrittura collocandosi "alla periferia della città paranoico-critica," per dirla con Salvador Dali, nella coscienza che - iuxta Lacan - "l'inconscio è il discorso dell'Altro" e "lo stile è l'uomo cui ci si rivolge": de/costruendola, appunto. De/scrivendola. Il romance, in questa strategia di allestimento smantellante, resiste come sinopia. I Minima Existentialia che Valesio estrae dal magma eracliteo finiscono comunque per coagulare in sistema: e il sistema è, ovviamente, quello del linguaggio. La natura primaria dell'operazione valesiana è metonimica: nel senso che i vari "pezzi" vengono a disporsi anche spazialmente in un ordine di contiguità che realizza (mi sia concessa, per un istante, licenza di paronomasia) un puzzle sommamente arbitrario e sommamente rigido. La topografia del Regno consiste di dieci località tra le quali la prima e l'ultima hanno la stessa nominazione ("La via dei minimi"). Le altre sono, in progressione: "Le viste," "I caratteri," "Le grammatiche," "I luoghi" (con le periferie de "I teatri" e de "I congressi"), "Gli strumenti," "Le maniere" (con le periferie de "I paesi," "Le cibarie," "Le cautele"), "Il danaro," "I corpi." Le sequenze narrative vengono frequentemente spezzate dall'inserzione di elementi parentetici destinati a produrre, piú che un puro effetto di inciso, un suspense logicoemotivo. Il blocchetto tagliato dal laser del fuoco della narrazione appare cosí internamente crinato, condannato a una compattezza precaria, sempre prossimo alla frantumazione. Il punto di vista del narratore Valesio sta, come avrebbe detto Baudelaire, che di narrativa e di narratività se ne intendeva assai, "sulla punta di un 
ago." Di qui la bellezza di queste pagine, la loro acuminata fragilità, la loro precisione ossessiva. L'occhio dello scrittore risulta, in questo gioco altero, singolarmente penetrante e singolarmente dolce: talvolta dolce, direi, della furiosa tenerezza di un autore dotato della grazia crudele e autolesionistica di Scott Fitzgerald.

In un saggio particolarmente luminoso Lionel Trilling parla della "musica abituale della serietà di Fitzgerald." Io ho, poco fa, accennato alla grazia dello stile di Valesio. "La grazia è sempre minacciata". dice lo stesso Valesio in un momento del Regno doloroso. La difesa da questa minaccia è, per il Valesio odierno, l'ironia: come ho già detto un po' addietro. Ma l'ironia presuppone la serietà: anzi, la presuppone drammaticamente. Appunto, tra serietà estrema ed estrema ironia si consuma il dramma dei tre personaggi che trascorrono nel libro (Doriana, Leo, Aurelio) come spettri gentili $\mathrm{o}$, se si preferisce, lèmuri taglienti. Immortale, fra $\mathrm{i}$ tre, è soltanto Doriana. Gli altri due dispongono soltanto della forza della subalternità, che è per sua natura destinata a finire. Ma gli altri due sono lo sdoppiamento di uno solo: la sua permanente, evanescente scissione. Soltanto un amore furioso per la lingua quale quello che Valesio esprime, senza peraltro il minimo scialo, la minima forzatura, può aspirare alla ricomposizione di una materia tanto macerata e sfuggente. Si tratta, proprio in linea col progetto del Valesio dell'Ospedale di Manhattan, di un amore per cosí dire diacronico, che recupera e rende smalto a una quantità di parole desuete della nostra tradizione anche arcaica, immettendole con leggerezza nervosa nel tessuto di una scrittura mobile, puntigliosa e, in molti casi, tremenda. La tragedia dell'esistere può rivelarsi difatti - e Paolo Valesio ce lo rivela splendidamente - anche nel modo in cui ci si abbottona la giacca, o in quello in cui si riempie una scheda in biblioteca. E in questo, c'è una somiglianza, o una similitudine perfino atroce tra l'America e l'Europa, tra Fifth Avenue e Via Giulia. Gli States sono la metafora dell'Europa. L'Europa è la metonimia degli States. 
MICHELANGELO PICONE. "Vita nuova" e tradizione romanza. Padova: Liviana Editrice, 1979. Pp. 203.

Parlare della Vita nuova non è facile. La sua apparente esilità è invece già sintesi, parola piena e sapiente che saldamente struttura il suo significato seguendolo da radici già dense di storia. Chi le si accosta non può non avvertire che quanto la pagina mostra è un levigato e ermetico episodio conclusivo, il risultato di una complessa elaborazione che sfugge attraverso una fitta rete di ramificazioni semantiche. Se non si vuole lasciarla intatta e chiusa nelle sue insondabili ragioni, leggerla sarà decifrarla. Le ragioni, quelle del suo spessore semantico, sono uno "spazio" e una "profondità" appunto da sondare. La lettura preliminare occorre si tenda allora fino al codice cui il testo si rapporta, cercandovi la "chiave" della decifrazione. L'"analisi sulla tematologia cortese della Vita nuova" che M. Picone nel suo saggio intraprende, ripercorre un tracciato diacronico che ha l'incisività di un "itinerario conoscitivo": quello di tutta la poesia amorosa romanza fino a Dante che ne matura il senso, e quello stesso, interno, del Poeta e dell'opera che assume il peso e la profondità della tradizione, la vaglia e la sperimenta, facendone strumento del suo piú audace percorso di conoscenza. Amore, cui si tende lo sforzo semantico della cultura romanza orientata dalla metafisica cristiana, è anche un vocabolo: "luogo invariante," primo in quel gruppo di parole comuni agli idiomi romanzi che rivelano un'originaria unica ed edenica fonte, la lingua primigenia in contatto con Dio per la pregnanza dei suoi contenuti. Beatrice, alla fine del tragitto conoscitivo modellato sull'esperienza romanza, sarà identificata come puro simbolo speculante Dio, edenico nomen consustanziale alla res, volto metafisico della lingua che la nomina.

La preoccupazione del critico è dunque quella di riportare l'opera all'interno della tradizione poetica per interpretarla e valutarla secondo i parametri geneticamente ad essa piú consoni. Il "contesto poetico-ideologico" spiega la "genesi," lo "sviluppo strutturale" e infine il "significato profondo" degli elementi tematici e ne illumina l'intreccio sintagmatico all'interno del testo. Ma soprattutto, è tenendo saldo il codice che è possibile reperire gli elementi innovatori, la forza centrifuga che dilata il significato del codice stesso: capire, in definitiva, il senso dell'operazione dantesca, che è quella di fissare un "articolato complesso di fatti culturali" per svilupparli e trascenderli, superando la tradizione cortese sulle tracce del suo stesso cammino.

Il luogo privilegiato di una sintassi tematica è l'inventio: luogo retorico, dove la scelta e la variazione dei temi acquistano un peso semantico in virtú anche della loro qualità retorica. Se la guinizzelliana "dolcezza" d'Amore è per Dante la "fonte culturale" della nova matera della loda, il nodo che trattiene Guinizzelli lontano dalla "novità" dantesca è l' "utilizzazione solo metaforica" della potenza di Amore: l'amor Dei è soltanto la metafora che descrive l'amore terreno. Allo stesso modo, la differenza fra Cavalcanti e Dante è quella tra un limite e una progressione: il limite di 